\title{
DIGITALLY PROGRAMMABLE ACTIVE-R FUNCTION GENERATOR
}

\author{
MUHAMMAD TAHER ABUELMA'ATTI \\ King Fahd University of Petroleum and Minerals, Box 203, Dhahran 31261, Saudi Arabia \\ (Received December 15, 1993; in final form February 22, 1994)
}

\begin{abstract}
A new digitally programmable active- $\mathrm{R}$ function generator circuit is presented. The circuit uses four operational amplifiers, two operational transconductance amplifiers, and resistors. The circuit can provide sinusoidal, square, and triangular waves with digitally programmable frequency. The frequency is linearly proportional with the digital input.
\end{abstract}

\section{INTRODUCTION}

Because external capacitors are eliminated altogether, active- $\mathrm{R}$ filters and oscillators using resistors only are very attractive for monolithic IC fabrication. This technique has been successfully used for implementing sinusoidal oscillators and square wave generators. ${ }^{1-3}$ However, its use for implementing function generators, simultaneously generating sinusoidal, square, and triangular waves has not been reported in the open literature.

The purpose of this paper is to present a novel active- $R$ function generator. The possibility of obtaining a digitally programmable frequency of oscillation will be explored.

\section{PROPOSED CIRCUIT}

Consider the circuit shown in Fig. (1). Let the open-loop gain of the operational amplifier be represented by the single-pole model given by

$\mathrm{A}(\mathrm{s})=\frac{\mathrm{A}_{0} \omega_{\alpha}}{\mathrm{s}+\omega_{\alpha}}$

where $A_{0}$ is the dc gain, $\omega_{\alpha}$ is the corner frequency, and $B=A_{0} \omega_{\alpha}$ is the gainbandwidth product of the operational amplifier. Direct analysis shows that the circuit formed by the operational amplifier OA3 and resistor $\mathrm{R}_{3}$ and the circuit formed of the operational amplifier OA4 and resistor $\mathrm{R}_{4}$ can be replaced by the series combinations $\mathrm{R}_{\mathrm{s}_{3}}-\mathrm{C}_{\mathrm{s}_{3}}$ and $\mathrm{R}_{\mathrm{s}_{4}}-\mathrm{C}_{\mathrm{s}_{4}}$ respectively, ${ }^{4.5}$ with

$\mathrm{R}_{\mathrm{s}_{\mathrm{i}}}=\mathrm{R}_{\mathrm{i}}, \quad \mathrm{i}=3,4$ 


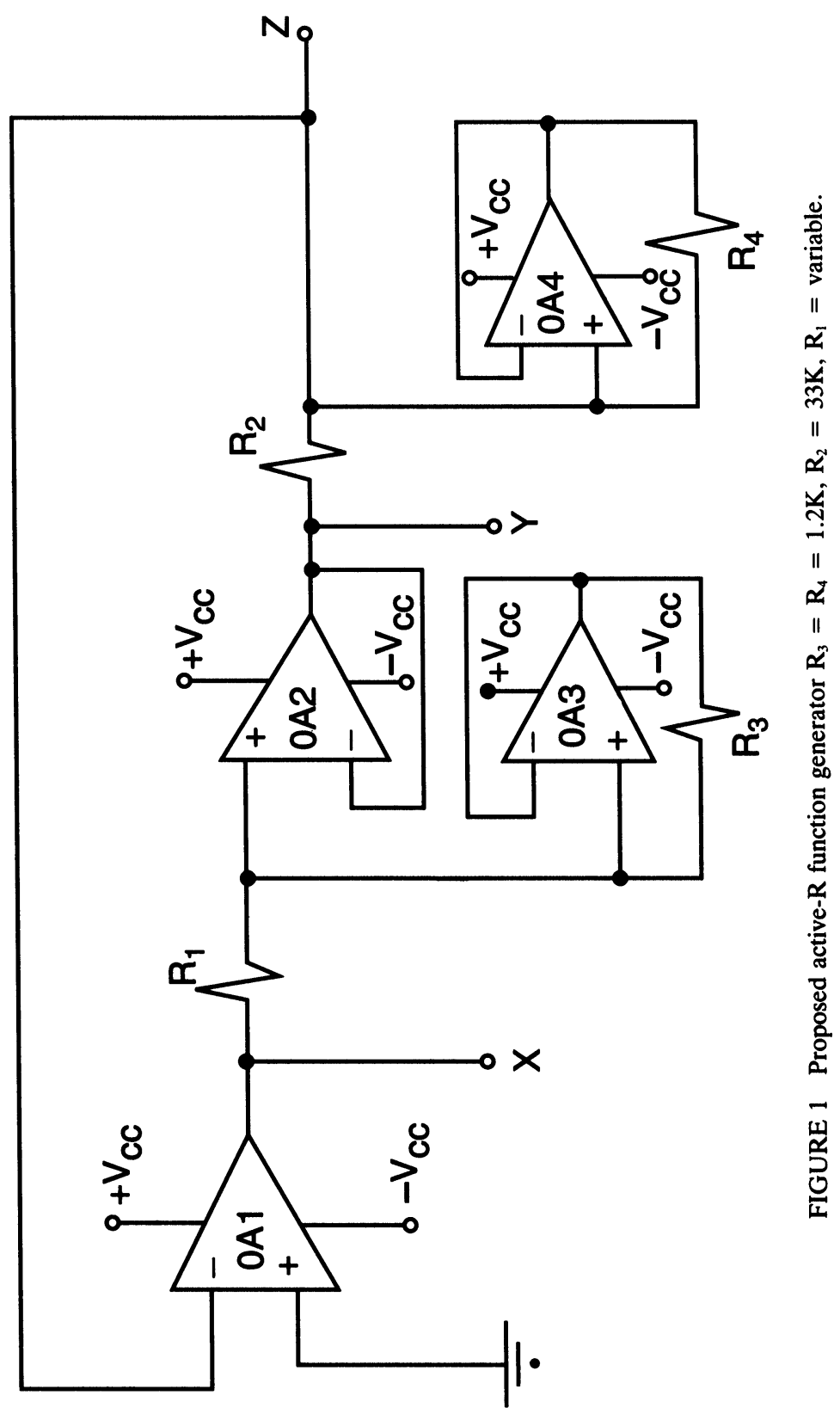


and

$\mathrm{C}_{\mathrm{s}_{\mathrm{i}}}=\frac{1}{\mathrm{BR}_{\mathrm{i}}}, \quad \mathrm{i}=3,4$

Therefore, the circuit of Fig. (1) can be reduced to the equivalent circuit shown in Fig. (2). A similar circuit is reported in Reference (6). Using this equivalent circuit, it is easy to show that the output at point $\mathrm{X}$ is a square wave with amplitude $=\left|\mathrm{V}_{\mathrm{CC}}\right|$; the operational amplifier OA1 is working as a comparator. This square wave will be integrated by the integrator formed of $R_{1}, R_{s_{3}}$ and $C_{s_{3}}$. Thus, the voltage at point $\mathrm{Y}$, with the operational amplifier $\mathrm{OA} 2$ working as a unity gain buffer, is a triangular wave of the form (see Appendix I)

$V_{Y}=\frac{V_{C C} R_{s_{3}}-V_{B} R_{1}}{\left(R_{1}+R_{s_{3}}\right)}+\frac{R_{1}\left(V_{C C}+V_{B}\right)}{C_{s_{3}}\left(R_{1}+R_{s_{3}}\right)^{2}} t$

Using eqn (4), the time $T_{1}$ required for the voltage at point $Y$ to change from $-V_{B}$ to $V_{B}$ is given by

$T_{1}=C_{s_{3}}\left(R_{1}+R_{s_{3}}\right) \frac{V_{B}\left(2 R_{1}+R_{s_{3}}\right)-V_{C C} R_{s_{3}}}{R_{1}\left(V_{C C}+V_{B}\right)}$

Similarly, the time required for the voltage at point $Y$ to change from $V_{B}$ to $-V_{B}$ is given by

$\mathrm{T}_{2}=\mathrm{T}_{1}$

Thus, the period of the triangular wave can be expressed as $T=T_{1}+T_{2}=2 T_{1}$ and the frequency of oscillation is given by

$\mathrm{f}=\frac{1}{\mathrm{~T}}=\frac{1}{2 \mathrm{C}_{\mathrm{s}_{3}}\left(\mathrm{R}_{1}+\mathrm{R}_{\mathrm{s}_{3}}\right)} \frac{\mathrm{R}_{1}\left(\mathrm{~V}_{\mathrm{CC}}+\mathrm{V}_{\mathrm{B}}\right)}{\mathrm{V}_{\mathrm{B}}\left(2 \mathrm{R}_{1}+\mathrm{R}_{\mathrm{s}_{3}}\right)-\mathrm{V}_{\mathrm{CC}} \mathrm{R}_{\mathrm{s}_{3}}}$

The lowpass filter $C_{2} R_{2}$ will convert the triangular wave at $Y$ into a sine wave at point $\mathrm{Z}$. This sine wave will be applied to the comparator $\mathrm{OA} 2$, thus producing a square wave at $X$ and the cycle will continue.

Combining eqns $(2,3$ and 7$)$ the frequency of oscillation can be expressed as

$$
f=\frac{B R_{3}}{2\left(R_{1}+R_{3}\right)} \frac{R_{1}\left(V_{C C}+V_{B}\right)}{V_{B}\left(2 R_{1}+R_{3}\right)-V_{C C} R_{3}}
$$

If we choose $R_{1} \gg R_{3}$ eqn (8) reduces to

$$
\mathrm{f} \simeq \frac{\mathrm{BR}_{3}}{2 \mathrm{R}_{1}}
$$


Thus, the frequency of oscillation will be inversely proportional to $R_{1}$. Also, from eqn (9) it is obvious that the frequency of oscillation is proportional to the gainbandwidth product, $\mathrm{B}$, of the operational amplifier.

Since the frequency of oscillation is inversely proportional to $R_{1}$ then by replacing $R_{1}$ by an electronically tunable resistor, the realization of an electronically tunable active-R function generator is feasible. Here we propose to use the circuit of Fig. (3) ${ }^{1}$ for implementing the electronically tunable resistor. The circuit uses two operational transconductance amplifiers and a single resistor. Direct analysis shows that

$\mathrm{I}_{\mathrm{B}_{2}}=\frac{\mathrm{V}-0.7}{\mathrm{R}_{\mathrm{B}}} \simeq \frac{\mathrm{V}}{\mathrm{R}_{\mathrm{B}}}$

$\mathrm{I}_{\mathrm{B}_{1}}=\mathrm{g}_{\mathrm{m}_{2}} \mathrm{~V}_{\mathrm{C}}$

$\mathrm{i}_{\text {out }}=\mathrm{g}_{\mathrm{m}_{1}} \mathrm{~V}_{\text {in }}$

where

$\mathrm{g}_{\mathrm{m}_{\mathrm{i}}}=\frac{\mathrm{I}_{\mathrm{Bi}}}{2 \mathrm{~V}_{\mathrm{T}}}, \quad \mathrm{i}=1,2$

Combining eqns (10)-(13), the equivalent resistance of the circuit of Fig. (3) will be

Req $=\frac{V_{\text {in }}}{i_{\text {out }}}=\frac{\left(2 V_{T}\right)^{2}}{V_{C}} \frac{R_{B}}{V}$

At room temperature, the thermal voltage $V_{T}$ equals $26 \mathrm{mV}$ and eqn (14) reduces to

$\operatorname{Req}=\frac{R_{B} / V}{369 V_{C}}$

From eqn (15) it is obvious that the equivalent resistance is inversely proportional to the control voltage $\mathrm{V}_{\mathrm{C}}$. By obtaining this voltage from the output of a digitalto-analog converter (DAC), the realisation of a digitally programmable electronically tunable resistor is feasible. Furthermore, by replacing the resistor $R_{1}$ of Fig. (1) by this digitally programmable resistor, the realisation of a digitally programmable, electronically tunable active- $R$ function generator is feasible. The frequency of oscillation will be linearly proportional with the digital input.

\section{EXPERIMENTAL RESULTS}

The function generator of Fig. (1) was built and tested using the uA741 operational amplifiers. Oscillation frequencies up to $10 \mathrm{KHz}$ were successfully obtained. To 


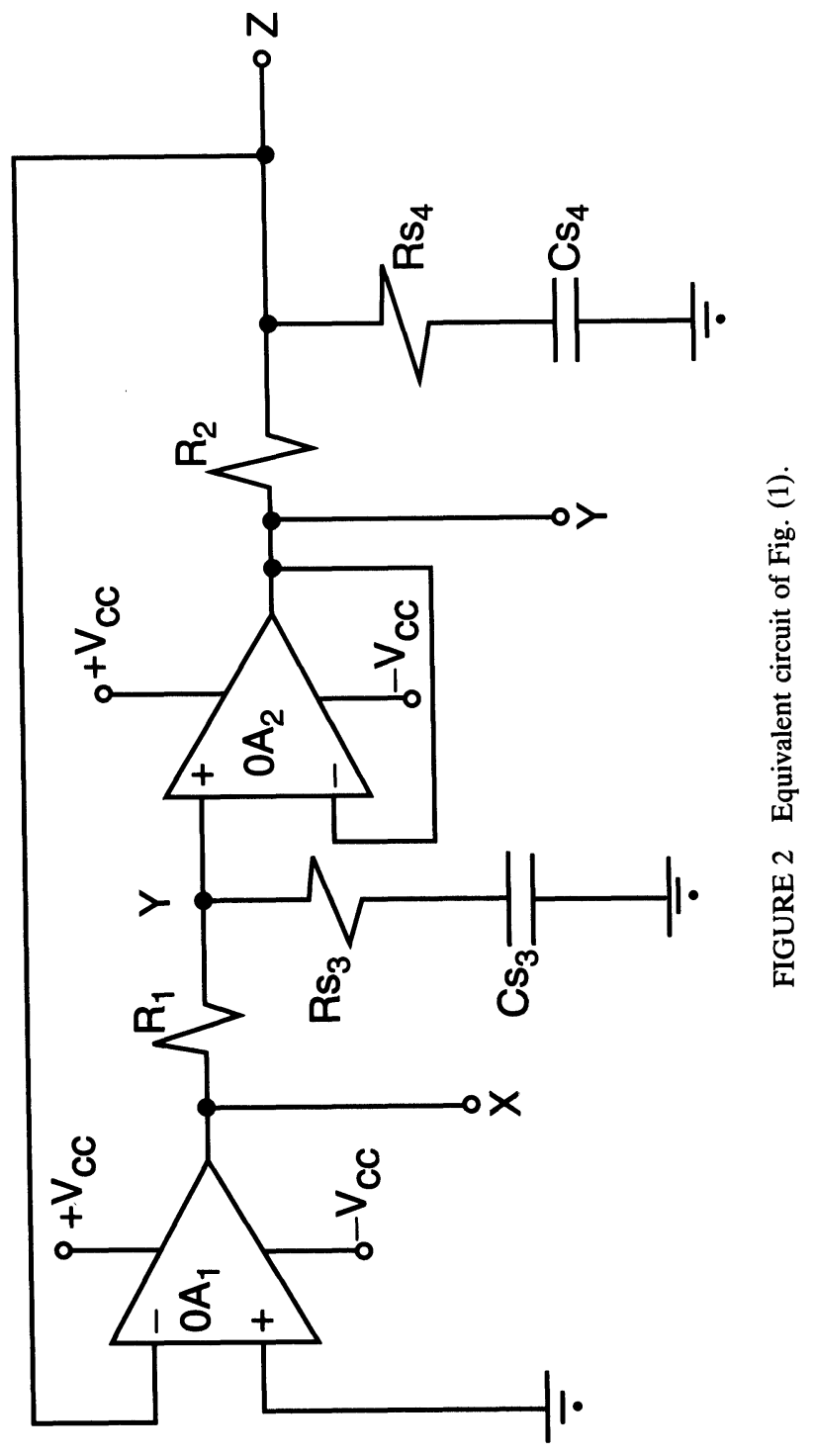




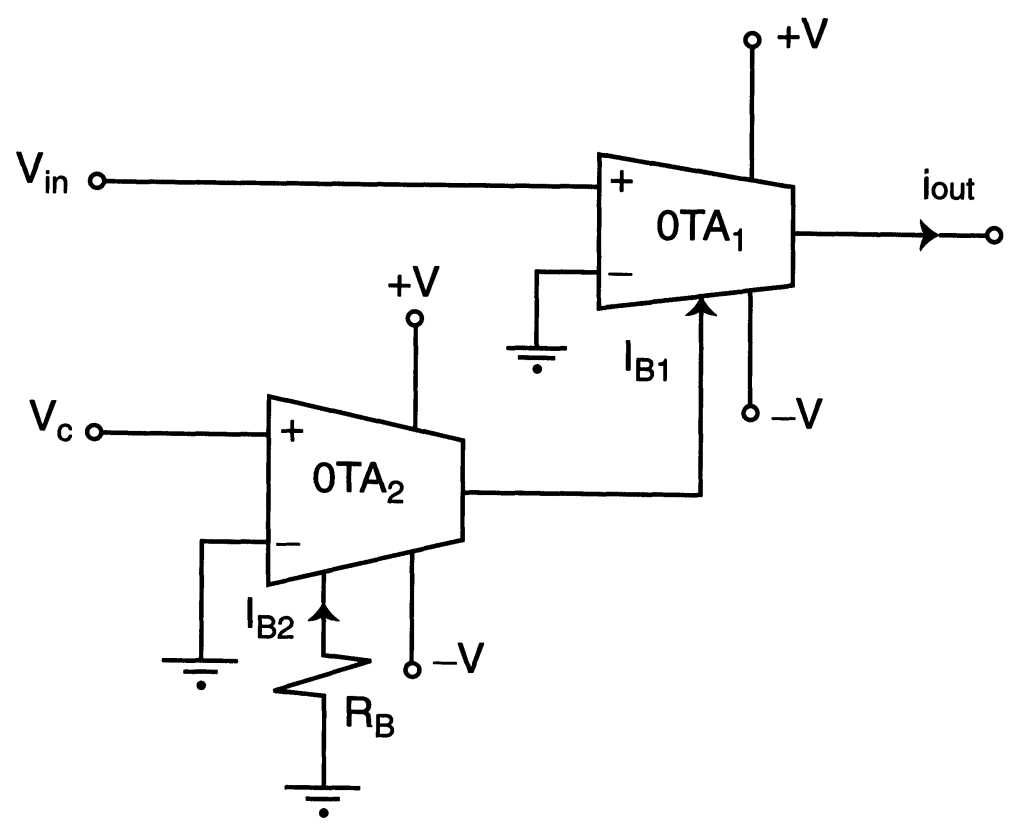

FIGURE 3 Proposed electronically tunable resistor.

investigate the feasibility of obtaining a digitally programmable, electronically tunable active-R function generator, the circuit of Fig. (4) was built and tested using 741 operational amplifiers and CA3080 operational transconductance amplifiers and a passive R-2R digital-to-analog converter. The digital input levels are $0 \mathrm{~V}$ for logic 0 and $5 \mathrm{~V}$ for logic 1 . Oscillations were successfully obtained and digitally controlled for digital inputs in the range 0000 to 1111 .

\section{CONCLUSION}

In this paper a new active- $R$ electronically tunable, digitally programmable function generator circuit has been presented. The main features of the circuit are the following:

1. It only uses resistors without recourse to any external capacitors. Therefore it is attractive for monolithic IC implementation.

2. It can be used with mini/microcomputer or microprocessor-based systems.

3. It is possible to obtain a linear relationship between the control voltage and the frequency of oscillation. 


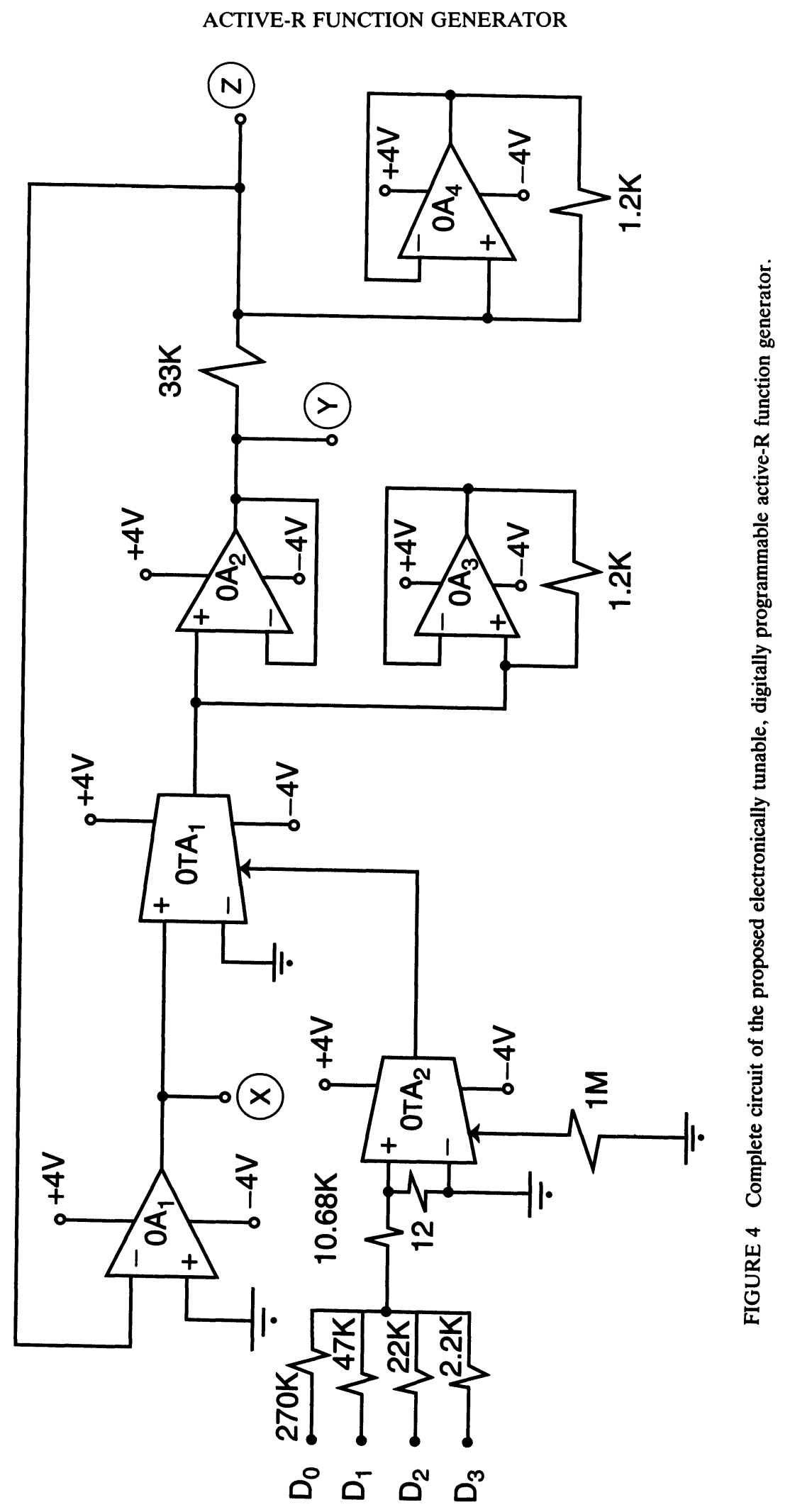




\section{REFERENCES}

1. M.T. Abuelma'atti, W.A. Almansouri, K.M. Alruwaihi, Digitally programmable active-R square generator, IEEE Transactions on Instrumentation and Measurement, Vol. 39, 1990, pp. 527-530.

2. M.T. Abuelma'atti and W.A. Almansouri, New voltage-controlled active-R oscillator, International Journal of Electronics, Vol. 61, 1986, pp. 255-259.

3. M.T. Abuelma'atti and W.A. Almansouri, Identification of two-amplifier active-R sinusoidal oscillators, Proceedings IEE, Vol. 134, Part G, 1987, pp. 137-140.

4. M.T. Ahmed, M.A. Siddiqi, M.T. Javed, A general synthesis technique for active-R networks, International Journal of Electronics, Vol. 54, 1983, pp. 417-425.

5. P.V. Ananda Mohan, Novel active filters using amplifier pole, Electronics Letters, Vol. 16, 1980 pp. 378-380.

6. M.T. Abuelma'atti and S.A. Al-Sayed, Function generator is digitally Programmable, Electronics World, Vol. 99, 1993, p. 411.

\section{APPENDIX I}

In this appendix the derivation of eqn (4) will be shown. Refer to Fig. (5) and consider an input voltage of the form shown in Fig. (6). Consider an instant of time when the input voltage changes from $-V_{C C}$ to $V_{C C}$. The capacitor now charges from $-V_{B}$ towards $V_{B}$ through the $\left(R_{1}+R_{s_{3}}\right) C_{s_{3}}$ combination. Thus, the voltage across the capacitor can be expressed as

$V_{C}(t)=-V_{B}+\left(V_{C C}+V_{B}\right)\left[1-\exp \left(-t /\left(C_{s_{3}}\left(R_{1}+R_{s_{3}}\right)\right)\right)\right]$

and the voltage drop across the resistor $R_{1}$ can be expressed as

$$
V_{R_{1}}(t)=R_{1} C_{s_{3}} \frac{d V_{C}}{d t}=\frac{R_{1}\left(V_{C C}+V_{B}\right)}{\left(R_{1}+R_{s_{3}}\right)} \exp \left(\frac{-t}{C_{s_{3}}\left(R_{1}+R_{s_{3}}\right.}\right)
$$

Thus, the voltage at point $\mathrm{Y}$ can be expressed as

$V_{Y}=V_{C C}-\left(V_{C C}+V_{B}\right) \frac{R_{1}}{R_{1}+R_{s_{3}}} \exp \left(-t /\left(C_{s_{3}}\left(R_{1}+R_{s_{3}}\right)\right)\right)$

For sufficiently small values of $t /\left[C_{s_{3}}\left(R_{1}+R_{s_{3}}\right)\right]$, that is sufficiently large time constants $C_{s_{3}}\left(R_{1}+R_{s_{3}}\right)$, eqn (A1) can be approximated by

$V_{Y}=\frac{V_{C C} R_{s_{3}}-V_{B} R_{1}}{\left(R_{1}+R_{s_{3}}\right)}+\frac{R_{1}\left(V_{C C}+V_{B}\right)}{C_{s_{3}}\left(R_{1}+R_{s_{3}}\right)^{2}} t$

Eqn (A2) shows that the voltage at point $Y$ will increase linearly with time. The time $T_{1}$ required for the voltage at point $Y$ to reach $V_{B}$ can be obtained from eqn (A2) by substituting $V_{Y}=V_{B}$ and $t=T_{1}$ and eqn (5) can be obtained. 

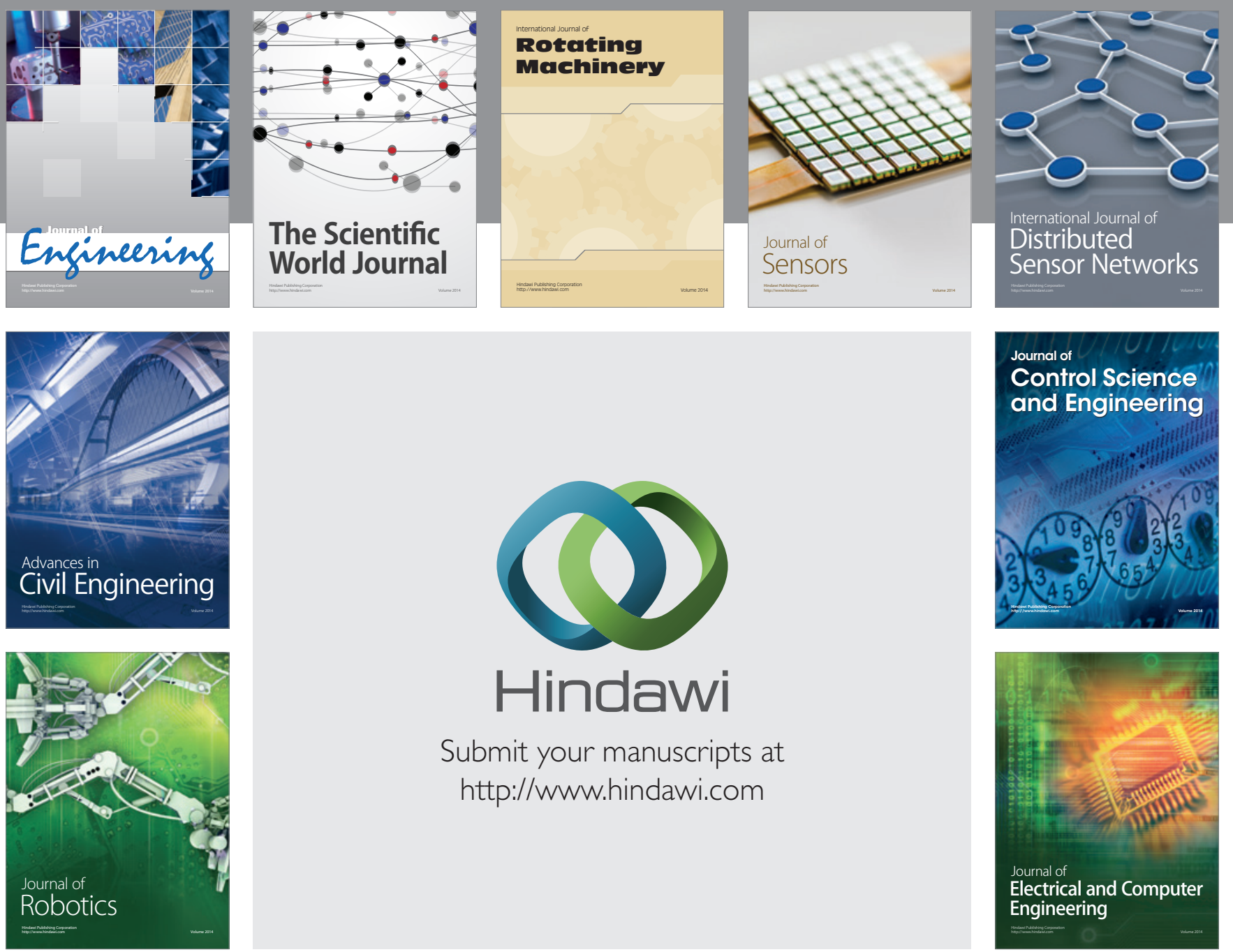

Submit your manuscripts at

http://www.hindawi.com
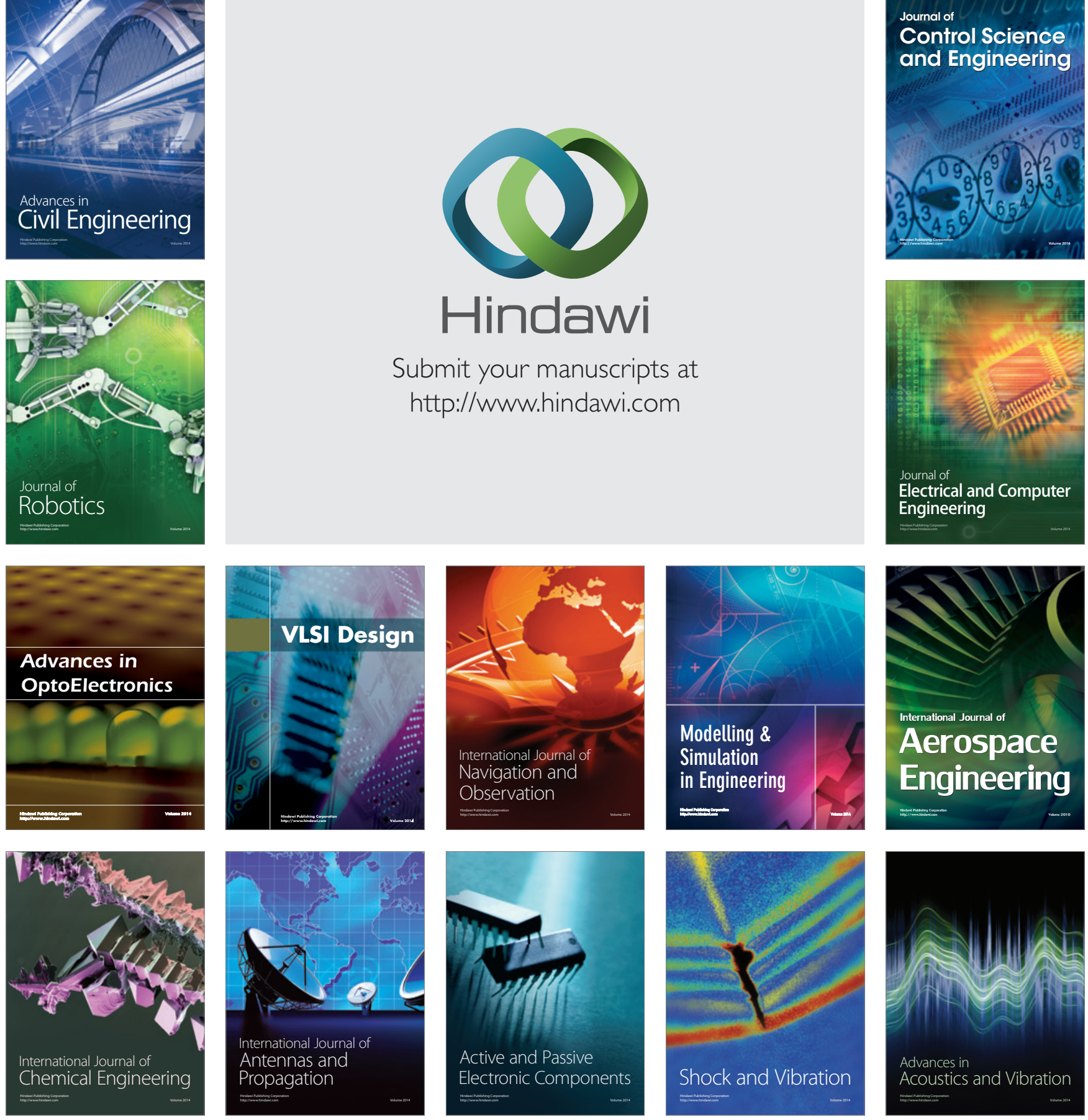